\title{
European Journal of Psychology Open
}

\section{New Beginnings, New Challenges}

\author{
Georgia Panayiotou ${ }^{1}$ and Samuel Greiff ${ }^{2}$ \\ ${ }^{1}$ Department of Psychology and Center for Applied Neuroscience, University of Cyprus, Nicosia, Cyprus \\ ${ }^{2}$ Department of Behavioural and Cognitive Sciences, University of Luxembourg, Esch-sur-Alzette, Luxembourg
}

It is with high aspirations, but also with a certain level of humility at the magnitude of the task, that we take on the Editorship of the European Journal of Psychology Open (EJPO), a new journal, launched by Hogrefe, as a continuation of the previously named Swiss Journal of Psychology. It was previously published together with the Swiss Society of Psychology and was originally founded by Jean Piaget in 1942. We would like to take up the long tradition of the journal and continue it together with EFPA. The European Federation of Psychologists Associations will incorporate the EJPO as an empirical journal to complement the existing organ, the European Psychologist, which focuses exclusively on the publication of review articles and integrative commentaries (Pawlik et al., 2020).

For these reasons, the need was identified to close the gap of an attractive outlet for empirical research, conducted throughout Europe but also around the world. As a result, we are happy to present EJPO.

The European Journal of Psychology Open highlights fresh and innovative research in psychology, brings to the fore the current questions, problems, and topics of concern to psychology, and the approaches and methods that psychologists use to tackle them. The overarching aim is to increase communication, understanding, and collaboration among European psychologists, both academic and applied. Unlike North America, where training programs in psychology are more or less uniform in structure, content, and to a great degree philosophy, European countries accredit programs at a national level, and programs differ greatly in duration, content, philosophy, and focus as well as language. This is the case for both undergraduate and graduate programs, both research-based and professional. A similar issue characterizes licensing criteria in psychology, which EFPA's EuroPsy certification aspired to address (Lunt, 2011). This potentially enriching diversity also poses barriers to communication and exchange of ideas as well as professional mobility. We hope that EJPO will become an arena through which researchers become aware of and have an opportunity to discuss diverse historical perspectives and traditions, cross-fertilize methodologies, and solve evolving scientific problems. Hence, EJPO aims to publish original, high-quality empirical papers from all fields of psychology. We anticipate that the submissions to the journal will put to the forefront findings and perspectives that both provide a map of current European research, its strengths, and its limitations and underscore topics that have received limited research attention but that are important for tackling contemporary scientific and practical problems. The journal welcomes papers from all parts of the world and expects to evolve into a truly international venue, presenting new ideas to take the field forward.

Another goal the journal aspires to, is to promote young talents. We wish to receive the scientific outputs and hear about the ideas and scientific endeavors of early career researchers (ECRs), as we expect these ideas best reflect current trends and cutting-edge research. While maintaining high standards of publication and rigorous peer-review processes, we are implementing ideas to encourage ECRs to submit their work and to benefit from the feedback received. After a transparent selection process, we have involved ECRs as the first five Associate Editors, all highly accomplished at an early stage in their careers, representing geographical diversity and different fields of psychology within Europe. We have also invited both eminent senior scholars and ECRs who represent young talent to become members of the Editorial Board, to provide valuable editorial and reviewing experiences to new researchers, while ensuring high standards of reviewing experience to our authors. We think that including ECRs in all realms of the publishing process serves to enrich the journal with fresh perspectives, energy, and curiosity, allowing us to hear and understand the concerns, fears, and disappointments 
of early career scholars, and to establish a humane, open, and respectful publishing environment.

As part of the reviews, it is our aim is to provide constructive and supportive feedback to all authors who submit their work, so that their submission can be improved, either for purposes of resubmission to EJPO or submission to an alternative outlet. In this way, we want to promote a culture of respect, scientific growth, and progress. Without a guarantee of publication, we nevertheless encourage ECRs with no prior publication experience to submit advanced drafts of manuscripts for feedback from our Editorial Board members, which they can then take into consideration when preparing the final manuscript for publication. We consider this an offer to help ECRs optimize their research and publishing skills, in a way few other journals in the field offer.

EJPO adheres to Open Science practices and is a signatory of the Transparency and Openness Promotion (TOP) Guidelines (see https://www.cos.io/our-services/ top-guidelines). We strongly encourage our authors to make their data, code, materials, and design available to the greatest degree possible (while recognizing the existence of ethical and legal limitations in some cases), while requiring them to be transparent about their choices regarding the level of open access selected. To encourage authors to implement open science practices, EJPO further awards Open Data (OD) and Open Materials (OM) badges when authors and papers meet the appropriate criteria. Although we do not currently publish preregistered reports, we encourage authors, especially early career scientists, to preregister their studies with the appropriate repositories and cite them in their submissions. Preregistration and open access encourage rigorousness, transparency, and replicability (Greiff et al., 2020; Simmons et al., 2021) - and combat bias and error.
We invite psychology scholars in all areas to join EJPO in this new venture.

\section{References}

Greiff, S., van der Westhuizen, L., Mund, M., Rauthmann, J. F., \& Wetzel, E. (2020). Introducing new open science practices at EJPA. European Journal of Psychological Assessment, 36(5), 717-720. https://doi.org/10.1027/1015-5759/a000628

Lunt, I. (2011). EuroPsy: The development of standards for highquality professional education in psychology. European Psychologist, 16(2), 104-110. https://doi.org/10.1027/1016-9040/ a000087

Pawlik, K., Silbereisen, R. K., Grob, A., Frensch, P., \& Lavallee, K. (2020). European Psychologist's 25th anniversary: Developments and perspectives. European Psychologist, 25(4), 231-236. https://doi.org/10.1027/1016-9040/a000428

Simmons, J.P., Nelson, L. D., \& Simonsohn, U. (2021). Preregistration: Why and how. Journal of Consumer Psychology, 31(1), 151-162. https://doi.org/10.1002/jcpy.1208

Prof. Georgia Panayiotou

Department of Psychology and Center for

Applied Neuroscience

University of Cyprus

Kallipoleos 75

1678 Nicosia

Cyprus

ejpoeditor@ucy.ac.cy

\section{Prof. Dr. Samuel Greiff}

Department of Behavioural and Cognitive Sciences

University of Luxembourg

2, avenue de l'Université

4366 Esch-sur-Alzette

Luxembourg 